\title{
Cloud Computing: Is a Step Ahead?
}

\author{
Mohammad Alamgeer, PhD \\ Assistant Professor \\ Department of Information System \\ King Khalid University \\ Kingdom of Saudi Arabia (KSA )
}

\author{
lqrar Ahmad \\ Research Scholar \\ Singhania University \\ Pacheri Bari, District Jhunjhunu \\ Rajasthan, India
}

\begin{abstract}
In last decade, cloud computing has been put forward, it can connect millions of computers to a big cloud. Cloud computing is not a new but an emerged model of business computing as an alternative to conventional computing. And it is becoming a development trend. This paper strives to compare and contrast cloud computing with supercomputers, cluster computing and grid computing, along with the challenges they face. This could help in bringing out the innovative ideas in the field and can be explored to the needs in the computing world. This could help in better understanding these models and to know how they differ from its related concepts, all in one go.
\end{abstract}

\section{Keywords}

Cloud computing; Grid computing; Cluster computing; Super computing

\section{INTRODUCTION}

Before a decade supercomputer was the leader in the field of computing. But due to some of the problems faced in the different field of engineering, science and business which could not be effectively dealt with using supercomputers they were replaced with clusters [1] with the objective of overcoming these problems and also provided a very cheap way for gaining access to potentially huge computing power. There was a need for high-performance computing at a lower cost which leads to cluster computing. High-performance computing could afford the significantly costly and dedicated supercomputers of the time. The emergence of cluster computing was driven by a number of academic projects, such as Beowulf [2]. Availability of powerful computers and highspeed network technologies and the popularity of the Internet has changed the way of using computers.

Grid computing started in academia at the last decade of 20th century with a purpose to facilitate users to remotely utilize idle computing power within other computing centers when the local one is busy [2]. Grid computing is a network that is not in the same place but distributed resources such as peripherals devices, computers, routers, switches, connectors and data. Its resources may be owned by different organizations. Grid can be viewed as a special type of middleware that enable sharing and manage grid components based on user requirements and resource attributes. You may have wondered about cloud computing as compared to grid computing. Cloud computing, is a kind of computing model that came into existence at the first decade of 21 st century. Cloud computing platforms are growing day by days. Cloud computing moves forward one step further with on-demand resource provisioning. This eliminates the need to overprovision in order to meet the demands of millions of users. A user can get service from a full computer infrastructure through the Internet. This kind of service is known as Infrastructure as a Service (IaaS). For example Internet-based services such as storage and databases are part of the IaaS. Other types of services on the Internet are Platform as a Service (PaaS) and Software as a Service (SaaS). PaaS offers partial or full application development that users can access, while SaaS provides a complete application, such as Enterprise Resource Management through the Internet [3, 4].

The main aim of cloud computing is to shift the computing done from the local computer into the network [5]. This makes the enterprise use the resource which includes storage, applications, network, server, services and so on that is required without huge investment on its purchase, implementation, maintenance rather use it for other significant purpose. Resources are requested on-demand without any prior reservation. Hence removes over provisioning and improves resource utilization. In this paper we bring out a complete comparison of the four computing models.

\section{OVERVIEW}

\subsection{Super Computing}

Supercomputers were introduced in the 1960 s by Seymour Cray at Control Data Corporation (CDC), Cray Research and subsequent companies bearing his name or monogram. While the supercomputers of the 1970s used only a small numbers of processors, in the 1990s machines with thousands of processors began to appear and, at the end of the 20th century, massively parallel supercomputers with tens of thousands of "off-the-shelf" processors were the norm [6, 7]. As of November 2013, China's Tianhe-2 supercomputer is the fastest in the world at 33.86 petaFLOPS. Systems with a large numbers of processors generally take one of two paths: in distributed computing, a large number of discrete computers like laptops distributed across a network devote some or all of their time to solving a common problem; each individual computer receives and completes many small tasks, reporting the results to a central server which integrates the task results from all the clients into the overall solution. In another approach, a massively number of dedicated processors are placed in close proximity to each other like computer cluster; this saves considerable time moving data around and makes it possible for the processors to work together rather than on separate tasks $[8,9]$. Supercomputers play a vast role in the field of computational science, and are used for a wide range of computationally intensive tasks in various fields, including quantum mechanics, weather forecasting, climate research, oil and gas exploration, molecular modeling such as computing the structures and properties of chemical compounds and physical simulations such as simulations of the early moments of the universe, airplane and spacecraft aerodynamics, nuclear fusion etc) [10].

\subsection{Cluster Computing}

A cluster is a collection of parallel or distributed computers which are interconnected using high-speed networks, such as gigabit Ethernet, Myrinet and Infiniband. They work together 
in the execution of data intensive tasks that would be not feasible to execute on a single computer. They are used for high availability purpose as they maintain redundant nodes which are used to provide service when system components fail. Clusters are used mainly for high availability, loadbalancing and compute purpose. When multiple computers are linked together in a cluster, they share computational workload as a single virtual computer. From the users point of view they are multiple machines, but they function as a single virtual machine. The user's request are received and distributed among all the standalone computers to form a cluster. This results in balanced computational work among different machines, improving the performance of the cluster systems.

\subsection{Grid Computing}

Grid computing is a method of distributed computing. It includes location and organization, software and hardware to provide unlimited power. Grid provides a series of distributed computing resource through LAN or WAN. To the terminal user of application, as is he is using a super virtual computer. This idea will realize safe and coordinate resource sharing among person, organization and resources, and will create virtual and dynamic organization. Its name is to make anyone in the grid can cooperate and access each other's information. But cloud computing is better. It has many advantages over grid computing in many ways. Computing grids are conceptually not unlike electrical grids. The aim of a computing grid, like that of the electrical grid, is to provide users with access to the resources they need, when they need them. In an electrical grid, wall outlets allow us to link to an infrastructure of resources that generate, distribute. And bill for electricity. When you connect to the electrical grid, you don't need to know where the power plant is or how the current gets to you. Grid computing uses middleware to coordinate disparate IT resources across a network, allowing them to function as a virtual whole [11]. Grids define two distinct but parallel goals: providing remote access to IT assets and aggregating processing power. The most obvious resource included in a grid is a processor but grids also contain datastorage systems, applications, sensors, and other resources. Many grids are used in the sciences such as chemistry, biology, physics and mathematicians have also begun working with grid computing. Grid technology has the potential to significantly impact other areas of study with massive computational requirements, such as urban planning [12]. Another important area for the technology is animation, which requires heavily amounts of disciplines. By making resources available to students, these communities are able to effectively model authentic disciplinary practices.

\subsection{Cloud Computing}

A large-scale distributed computing paradigm that is driven by economies of scale, in which a pool of abstracted virtualized, dynamically-scalable, managed computing power, storage, platforms, and services are delivered on demand to external customers over the Internet [13]. There are some important points in this definition. First, Cloud Computing is a specialized distributed computing paradigm; it differs from traditional ones in that it can be encapsulated as an abstract entity that delivers different levels of services to customers outside the Cloud it is driven by economies of scale it is massively scalable the services can be dynamically configured and delivered on demand [14].

The following three main types of services that can be offered by the cloud:
- Infrastructure as a Service (IaaS): Products offered via this mode include the remote delivery of a full computer infrastructure (e.g., virtual computers, servers, storage devices, etc) [15].

- Platform as a Service (PaaS): Under this layer, one needs to remember the traditional computing model where each application managed locally required hardware, an operating system, a database, middleware, Web servers, and other software. One also needs to remember the team of network, database, and system management experts that are needed to keep everything up and running. With cloud computing, these services are now provided remotely by cloud providers under this layer.

- Software as a Service (SaaS): In this layer applications are delivered through the medium of the Internet as a service. Instead of installing and maintaining software, you simply access it via the Internet, freeing yourself from complex software and hardware management [16]. This type of cloud service provides a complete application functionality that ranges from office type applications to programs such as those for enterprise-resource management.

\section{CHALLENGES IN SUPER, CLUSTER, GRID AND CLOUD COMPUTING}

Each model has some common challenges needs to convert challenges into opportunities. We highlight some of the challenges of the computing models considered in this paper.

\subsection{Challenges in the Super Computing}

The research challenges faced in grids include:

- Heterogeneity: To develop a wide area data intensive programming and scheduling framework in heterogeneous set of resources.

- Programming: The low-coupling among the nodes and the distributed nature of processing make the programming of applications more complex.

- $\quad$ Elasticity: The variance in real-time response time when the number of service requests changes dramatically.

- Scalability: To fulfill the additional requirements of a resource that effecting the performance of the system.

\subsection{Challenges in the Cluster Computing}

The research challenges of cluster computing are as follows:

- Middleware: To produce software environments that provides an image of a single system, rather than a collection of independent computers.

- Program: The applications that run on the clusters must be explicitly written which incorporates the division of tasks among the nodes; also the communication among them should be taken care of.

- Elasticity: The variance in real-time response time when the number of service requests changes dramatically.

- Scalability: To fulfill the additional requirements of a resource that effects the performance of the system. 


\subsection{Challenges in grid computing}

The research challenges faced in grids include:

- Dynamicity: Resources in grid are owned and managed by more than one organization which may enter and leave the grid at any time causing massive load on the grid.

- Administration: To create a unified resource pool, a massive system administration load is raised along with other maintenance work to coordinate local administration policies with global ones.

- Development: Problems are concerned with ways of writing software to run on grid-computing platforms, which includes decomposing and distributing to processing elements, and then assembling solutions.

- Accounting: developing the methods to support different accounting infrastructure, economic model and application models that can face with the tasks that communicate frequently and are interdependent.

- $\quad$ Programming: The low-coupling between nodes and the distributed nature of processing make the programming of applications over grids more complex.

\subsection{Challenges in the cloud computing}

The challenges of cloud computing includes the following:

- Dynamic scalability: The compute nodes are scaled up and down dynamically by the application according to the response time of the user's queries. The scheduling delays involved are real concern which leads to the need of effective and dynamic load management system.

- Multi-tenancy: The amount of bandwidth allocated will reduce to each application when the number of applications running on the same compute node increases which may lead to performance degradation.

- Querying and access: Scalable provenance querying and secure access of provenance information are big problems.

- Standardization: The integration and interoperability of all the services and application is a challenge because of every organization has own APIs and protocols used which makes the user data or vendor lock-in.

- Reliability and fault-tolerance: Compute failures helps in developing a reliable system therefore tools are required for testing the application against the fault tolerance

- Security and Privacy: Security and privacy are most important concerned that user has no idea where data is stored and who will use. There are more hackers than developers.

- Power: Due to offering many types of services to meet the needs of users, enormous amount of power is consumed. For managing the resources an application is required.

\section{COMPARISON OF SUPER, CLUSTER, GRID AND CLOUD COMPUTING}

This section highlights an end-to-end comparison among the different computing models. It represented in a tabular form in table I which shows how the features of cloud computing model are differ the others models.

Table 1. Comparison of super, cluster, grid and cloud computing

\begin{tabular}{|l|c|c|c|c|}
\hline & $\begin{array}{c}\text { Super } \\
\text { computers }\end{array}$ & Clusters & grids & Clouds \\
\hline Reliability & Low & Low & High & $\begin{array}{c}\text { Very } \\
\text { High }\end{array}$ \\
\hline Security & VeryHigh & VeryHigh & Low & $\begin{array}{c}\text { Very } \\
\text { Low }\end{array}$ \\
\hline $\begin{array}{l}\text { Resource } \\
\text { Handling }\end{array}$ & Centralized & Centralized & Distributed & Both \\
\hline Business Model & No & No & No & Yes \\
\hline Interoperability & Yes & Yes & Yes & Half \\
\hline Coupling & Very & Strong & strong & Loose \\
\hline Virtualization & Low & Half & Half & High \\
\hline Switching Cost & VeryLow & Low & Low & High \\
\hline $\begin{array}{l}\text { Data Locality } \\
\text { Exploited }\end{array}$ & No & No & No & Yes \\
\hline Expensine & Yes & Yes & Yes & No \\
\hline Multitenancy & No & No & Yes & Yes \\
\hline Task Size & Single & Single & Single & Small \& \\
Large & Large & Large & Medum \\
\hline Heterogeneity & No & No & Yes & Yes \\
\hline Standardized & Yes & No & Yes & Yes \\
\hline Scalable & No & No & Half & Yes \\
\hline & & & & Yes \\
\hline
\end{tabular}

\section{WHY CLOUD COMPUTING?}

The main aim of cloud computing is to shift the computing done from the local computer into the network. This makes the enterprise use the resource which includes storage, applications, network, server, services and so on that is required without huge investment on its purchase, implementation, maintenance rather use it for other significant purpose. Resources are requested on demand without any prior reservation. Hence removes over provisioning and improves resource utilization. Advances in technology offer new opportunities in enhancing teaching and learning so cloud computing has a significant impact on the e-learning environment. The features and advantages of Cloud computing in compare to other models are as follows [17]: 
1. Super computing power: It makes the support of varies of applications convenient and fast to retrieve the powerful computing and storage resources.

2. High resources availability: It provides real-time configuration, allocates resources on-demand and improves the usage rate of available resources.

3. Security: Cloud manages information by a team of professional. It facilitates strict rights management strategy during accessing data.

4. Virtualization: Each application deployment environment and physical platform is managed, expensed, migrated, and backup through virtualization platform.

5. Crash recovery: If your devices are crashes, no data lost because this data is stored in the cloud [18].

6. Convenience: It is not necessary to download software and data. You can access cloud services anytime and anywhere through Internet [19]. Cloud allows students to work from multiple Places such as college, home, office etc, find their files and edit them. Also browser-based applications can also be accessed through various devices such as mobile, tablet, laptop and desk top computers etc [20].

7. Economic efficiency. There is no need buying expensive hardware for cloud computing and then the education platform could be easily established. Get the most out of efficiency of hardware and software. It provides a low cost solution to academic institutions for their researchers, faculty, clerck and students [21].

8. Flexibility: Cloud computing allows user to dynamically scale as demands vary. Scale infrastructure to take full advantage of investments [22].

\section{CONCLUSION}

In this paper, we presented compare and contrast of Cloud Computing with Supercomputers, Cluster Computing and Grid Computing, along with the challenges they face. The issues and challenges related to these computing models are highlighted. Such a comparison in different perspectives will make easy to understand the computing models since the features of these computing models seems to be similar conceptually. This comparison shows that cloud computing a step ahead than other computing models. We think it is more likely that all other technologies will merge into cloud computing. And we think over the next couple of years more than 90 percent of the computation we are doing could be cloud-based for the become one global infrastructure for information, knowledge, computation and communication. More users will be switching to cloud computing and the value added services will be a key factor rather than the price factor for selection of cloud service providers.

\section{REFERENCES}

[1] "Cluster Computing", http://en.wikipedia.org/wiki /Cluster computing.

[2] Beowulf, http://www.beowulf.org

[3] http://searchcloudcomputing.techtarget.com/sDefinition/ 0,sid201_gci1287881,00.html

[4] http://www.ibm.com/developerworks/library/wacloudgrid/

[5] I. Foster, C. Kesselman, S. Tuecke, "The Grid: Blueprint for a New Computing Infrastructure", Intl. Jr. of Supercomputer Applications, 15(3),2001.

[6] Hoffman, Allan R.; et al. (1990). Supercomputers: directions in technology and applications. National Academies. pp. 35-47. ISBN 0-309-04088-4.

[7] Hill, Mark Donald; Jouppi, Norman Paul; Sohi, Gurindar (1999). Readings in computer architecture. pp. 40-49. ISBN 1-55860-539-8

[8] Prodan, Radu; Fahringer, Thomas (2007). Grid computing: experiment management, tool integration, and scientific workflows. pp. 1-4. ISBN 3-540-69261-

[9] DesktopGrid

[10] http://en.wikipedia.org/wiki/Supercomputer

[11] http://www.iturls.com/English/TechHotspot

[12] EMC 2008 Annual Overview Releasing the power of information, http://www.emc.com /digital_universe

[13] ]https://resources.sei.cmu.edu/asset_files/WhitePaper/201 0_019_001_29025.pdf

[14] J. Silvestre. "Economies and Diseconomies of Scale," The NewPalgrave: A Dictionary of Economics, v. 2, pp. 80-84, 1987.

[15] The four benefits of cloud computing, Sina science and technology,http://www.cloudcomputingchina.cn/Article/jh/200811/218.html,November 13, 2008

[16] On the future technology trends: the development of cloud computing security, http://stor.zol.com.cn/128/1288536.html, April 13, 2009 (reproduced)

[17] Zhang Guoli and Liu Wanjun, The Applied Research of Cloud Computing Platform Architecture In the ELearning Area, Proceedings of the 2nd IEEE International Conference on Computer and Automation Engineering 2010, Singapore, pp. 356-359, February 26$28,2010$.

[18] Pocatilu, P., F. Alecu, et al. "Measuring the Efficiency of Cloud Computing for E-learning Systems", Romania January 2010 .

[19] http://www.dontwasteyourtime.co.uk, Benefits of Collaborative Learning. Jul 8 .By David Hopkins.

[20] A1-Zoube, M., S. A. EI-Seoud, et al. "Cloud Computing Based ELearning System". "International Journal of Distance Education Technologies (UDET)", 2010, S(2).

[21] http://www.rnicrosofi.com.Cloud computing For Education.

[22] T. E. "Effective Use Of Cloud Computing In Educational Institutions". January 5, 2010, Turkey, from Elsevier 